\title{
Mathieu Moonshine in the elliptic genus of K3
}

\section{Journal Article}

Author(s):

Gaberdiel, Matthias R.; Hohenegger, Stefan; Volpato, Roberto

Publication date:

2010-10

Permanent link:

https://doi.org/10.3929/ethz-b-000158838

Rights / license:

Creative Commons Attribution 4.0 International

Originally published in:

Journal of High Energy Physics 2010(10), https://doi.org/10.1007/JHEP10(2010)062 


\section{Mathieu Moonshine in the elliptic genus of $\mathrm{K} 3$}

\section{Matthias R. Gaberdiel, Stefan Hohenegger and Roberto Volpato}

Institut für Theoretische Physik, ETH Zurich, CH-8093 Zürich, Switzerland

E-mail: gaberdiel@itp.phys.ethz.ch, stefanh@itp.phys.ethz.ch, volpato@itp.phys.ethz.ch

ABSTRACT: It has recently been conjectured that the elliptic genus of K3 can be written in terms of dimensions of Mathieu group $\mathbb{M}_{24}$ representations. Some further evidence for this idea was subsequently found by studying the twining genera that are obtained from the elliptic genus upon replacing dimensions of Mathieu group representations by their characters. In this paper we find explicit formulae for all (remaining) twining genera by making an educated guess for their general modular properties. This allows us to identify the decomposition of all expansion coefficients in terms of dimensions of $\mathbb{M}_{24^{-}}$ representations. For the first 500 coefficients we verify that the multiplicities with which these representations appear are indeed all non-negative integers. This represents very compelling evidence in favour of the conjecture.

Keywords: Extended Supersymmetry, Conformal Field Models in String Theory

ARXIV EPRINT: 1008.3778 


\section{Contents}

1 Introduction 1

2 Elliptic genus and twining characters 3

3 Modular properties of the twining genera 5

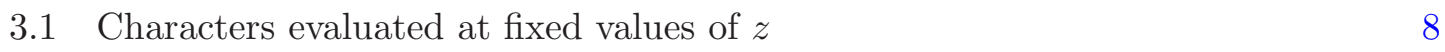

$\begin{array}{lll}3.2 & \text { Studying the modular forms of weight two } & 10\end{array}$

4 Decomposition into irreducible representations 11

5 Conclusions 12

$\begin{array}{ll}\text { A Definitions } & 15\end{array}$

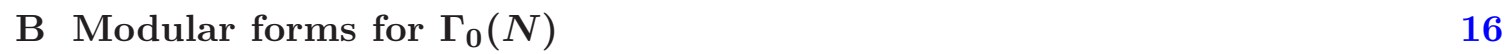

$\begin{array}{ll}\text { C The modular forms of weight two } & 19\end{array}$

\section{Introduction}

A few months ago Eguchi, Ooguri and Tachikawa [1] observed that the elliptic genus of K3 seems to involve representations of the largest of the Mathieu groups, $\mathbb{M}_{24}$. More specifically, they studied the expansion of the elliptic genus of K3 in terms of elliptic genera of $\mathcal{N}=4$ superconformal representations following [2], and noted that the expansion coefficients can be written in terms of dimensions of representations of $\mathbb{M}_{24}$. This intriguing observation is very reminiscent of a similar phenomenon usually referred to as 'Monstrous Moonshine', namely that the famous $J$-function has an expansion in terms of characters of the Virasoro algebra whose coefficients are dimensions of Monster group representations, as was first noted by McKay and Thompson.

In the context of Monstrous Moonshine, this observation was eventually explained by the construction of the so-called Monster conformal field theory $V^{\natural}$ [3], a self-dual conformal field theory at $c=24$ whose space of states is of the form

$$
V^{\natural}=\bigoplus_{n=0}^{\infty}\left(V_{n} \otimes \mathcal{H}_{h=n}^{\mathrm{Vir}}\right),
$$

where each $V_{n}$ is a representation of the Monster group, while $\mathcal{H}_{h}^{\text {Vir }}$ denotes the irreducible Virasoro representation with conformal weight $h$ and $c=24$. The $J$-function is then the partition function of $V^{\natural}$, and its Fourier coefficients are therefore sums of dimensions of 
irreducible Monster group representations. Furthermore, the automorphism group of $V^{\natural}$ is the Monster group.

One key observation that provided convincing evidence for (1.1) came from considering the so-called McKay-Thompson series [4]. These are obtained from the $J$-function upon replacing the expansion coefficients $A_{n}=\operatorname{dim}\left(V_{n}\right)$ by their corresponding characters, $\operatorname{Tr}_{V_{n}}(g)$, where $g$ is an element of the Monster group. ${ }^{1}$ It was shown by Conway \& Norton [5] that these McKay-Thompson series have nice modular properties under congruence subgroups of $\mathrm{SL}(2, \mathbb{Z})$. This is what one expects if they arise indeed from (1.1) since they are then equal to the 'twining character', i.e. the character with the insertion of the group element $g$, which has good modular properties based on standard orbifold arguments. For a review of these and other aspects of Monstrous Moonshine see e.g. [6].

By analogy to (1.1) the observation of [1] suggests that the states that contribute to the elliptic genus of K3 have the structure

$$
\mathcal{H}^{\mathrm{BPS}}=\bigoplus_{n}\left(H_{n} \otimes \mathcal{H}_{n}^{\mathcal{N}=4}\right),
$$

where the sum runs over all irreducible $\mathcal{N}=4$ representations that contribute to the elliptic genus, while each $H_{n}$ is a $\mathbb{M}_{24}$ representation. In order to test this idea it is then again natural to consider the 'twining genera' $\phi_{g}(\tau, z)$, where one replaces $\operatorname{dim}\left(H_{n}\right)$ by the corresponding character $\operatorname{Tr}_{H_{n}}(g)$, with $g \in \mathbb{M}_{24}[7,8]$. Unfortunately, the dimensions of the irreducible representations of $\mathbb{M}_{24}$ are rather small, and the decomposition of the expansion coefficients of the elliptic genus of $\mathrm{K} 3$ in terms of dimensions of $\mathbb{M}_{24}$ representations could only be guessed reliably for the first few coefficients. However, the elliptic genus of K3 is a weak Jacobi form of index one and weight zero [9], and one expects that the twining genera should have similar properties. More specifically, if $g \in \mathbb{M}_{24}$ has order $N$, then the corresponding twining genus $\phi_{g}(\tau, z)$ should transform as a weak Jacobi form of index 1 and weight 0 under the congruence subgroup $\Gamma_{0}(N)$, possibly up to a multiplier system.

In $[7,8]$ this assumption about the modular properties of the twining genera, together with the explicit knowledge of the first few coefficients, was used in order to determine some of them explicitly. The fact that these two constraints were compatible was already a fairly non-trivial consistency check on the proposal of [1]. Furthermore, for group elements that enjoy an interpretation as an automorphism symmetry of $\mathrm{K} 3$ at a suitable point in moduli space, one could calculate the corresponding twining genera directly (see in particular [10]). In this way some of these formulae could be confirmed independently [7].

In this paper we shall complete the analysis of $[7,8]$ by finding explicit formulae for the remaining conjugacy classes. ${ }^{2}$ In particular, we shall make a precise proposal for the structure of the multiplier system that appears in the modular transformation formula, see (3.8) and table 1 below. We can then follow again the strategies outlined in [8] and [7], respectively, to determine the twining genera, using the explicit knowledge of the first few

\footnotetext{
${ }^{1}$ These series can be calculated provided one knows how to write the Fourier coefficients of the $J$-function as $A_{n}=\operatorname{dim}\left(V_{n}\right)$, but do not require any additional knowledge.

${ }^{2}$ After posting the first version of this paper on the arXiv, we were informed by Tohru Eguchi that they have independently obtained explicit formulae for these twining genera which agree with ours, see [24].
} 
terms. The fact that this procedure is successful is again a fairly non-trivial consistency check of the proposed structure (1.2).

The knowledge of all twining genera also leads to another, highly constraining, consistency check. If (1.2) holds and if, for each $g$, our explicit formula for $\phi_{g}$ really corresponds to the trace over $\mathcal{H}^{\mathrm{BPS}}$ with the insertion of $g$, then its coefficients must be equal to $\operatorname{Tr}_{H_{n}}(g)$ for all $n$. But knowing these traces for all classes $g$ is sufficient to identify unambiguously $H_{n}$ as a (possibly reducible) representation of $\mathbb{M}_{24}$. Thus assuming that (1.2) holds, we can deduce the decomposition of $H_{n}$ into irreducible $\mathbb{M}_{24}$ representations for all $n$. We have calculated the multiplicities explicitly up to order 500 - the results up to order 30 are tabulated in table 4 - and they are all indeed non-negative integers. The fact that this decomposition works out, i.e. that each $H_{n}$ can be written as a direct sum of irreducible representations (with integer multiplicities) is then a highly non-trivial check of (1.2); in fact, we would in some sense prove the proposal of [1] if we could show that all of these multiplicities are indeed non-negative integers.

The paper is organised as follows. In section 2 we briefly review some of the properties of the elliptic genus of K3, as well as the proposal of [1]. The modular properties of the twining genera are explained in detail in section 3. In particular, by analogy with the situation for the McKay-Thompson series of Monstrous Moonshine, we make a specific proposal for the structure of the multiplier system. This proposal is then subsequently verified in sections 3.1 and 3.2. In section 3.1 we adopt the strategy of [8] and consider the twining genus at specific values of $z$, finding explicit formulae for all remaining cases. Section 3.2, on the other hand, employs the method of [7] to write the twining genus in terms of a modular form of weight two; this allows us to check some of the modular properties of the twining genera fairly directly, but the analysis is quite complicated and the details are described in appendix B and C. Finally, we explain in section 4 how the knowledge of all twining genera determines the decomposition of the coefficients in terms of $\mathbb{M}_{24}$ representations, and give the explicit results in table 4 . We close with some comments and speculations in section 5 .

\section{$2 \quad$ Elliptic genus and twining characters}

The elliptic genus of an $\mathcal{N}=2$ superconformal algebra is defined by

$$
\phi(\tau, z)=\operatorname{Tr}_{\mathcal{H}_{\mathrm{RR}}}\left(q^{L_{0}-\frac{c}{24}} e^{2 \pi i z J_{0}}(-1)^{F} \bar{q}^{\bar{L}_{0}-\frac{\bar{c}}{24}}(-1)^{\bar{F}}\right),
$$

where $q=e^{2 \pi i \tau}$, and the trace is taken in the RR sector. For the right-movers (whose modes are denoted by a bar), only the ground states contribute, and hence the above expression is in fact independent of $\bar{q}$. As is well known [9], the modularity properties of conformal field theory together with spectral flow invariance and unitarity imply that the elliptic genus is a weak Jacobi form of index $m=\frac{c}{6}$ and weight 0 [11]. A weak Jacobi form $\phi(\tau, z)$ of weight $w$ and index $m \in \mathbb{Z}$ is a function $\phi$ of $(\tau, z) \in \mathbb{H} \times \mathbb{C}$, where $\mathbb{H}$ is the upper 
half-plane. It is characterised by the transformation properties

$$
\begin{aligned}
\phi\left(\frac{a \tau+b}{c \tau+d}, \frac{z}{c \tau+d}\right) & =(c \tau+d)^{w} e^{2 \pi i m \frac{c z^{2}}{c \tau+d}} \phi(\tau, z) & \left(\begin{array}{ll}
a & b \\
c & d
\end{array}\right) \in \operatorname{SL}(2, \mathbb{Z}), \\
\phi\left(\tau, z+\ell \tau+\ell^{\prime}\right) & =e^{-2 \pi i m\left(\ell^{2} \tau+2 \ell z\right)} \phi(\tau, z) & \ell, \ell^{\prime} \in \mathbb{Z},
\end{aligned}
$$

and has a Fourier expansion

$$
\phi(\tau, z)=\sum_{n \geq 0, \ell \in \mathbb{Z}} c(n, \ell) q^{n} y^{\ell},
$$

where $y=e^{2 \pi i z}$ and $c(n, \ell)=(-1)^{w} c(n,-\ell)$. For the case of K3 that will concern us primarily in this paper, $m=1$ and the elliptic genus equals [12]

$$
\phi_{\mathrm{K} 3}(\tau, z)=2 y+20+2 y^{-1}+q\left(20 y^{2}-128 y+216-128 y^{-1}+20 y^{-2}\right)+\mathcal{O}\left(q^{2}\right) .
$$

It can be thought of as the partition function of the $\mathcal{N}=2$ half-BPS states of type II string theory on K3.

For the case of K3 the conformal field theory is actually $\mathcal{N}=4$ superconformal, and one can therefore write the elliptic genus in terms of the elliptic genera associated to $\mathcal{N}=4$ superconformal representations. It was observed in [1], following on from earlier work [2], that it can be written as

$$
\phi_{\mathrm{K} 3}(\tau, z)=24 \operatorname{ch}_{h=\frac{1}{4}, l=0}^{\mathcal{N}=4}(\tau, z)+\sum_{n=0}^{\infty} A_{n} \operatorname{ch}_{h=n+\frac{1}{4}, l=\frac{1}{2}}^{\mathcal{N}=4}(\tau, z),
$$

where $\operatorname{ch}_{h=\frac{1}{4}, l=0}^{\mathcal{N}=4}$ is the elliptic genus of the short $\mathcal{N}=4$ representation with $h=\frac{1}{4}$ and $l=0$ - see $[13,14]$ for an explicit formula — while

$$
\operatorname{ch}_{h, l=\frac{1}{2}}^{\mathcal{N}=4}(\tau, z)=q^{h-\frac{3}{8}} \frac{\vartheta_{1}(\tau, z)^{2}}{\eta(\tau)^{3}}
$$

is the elliptic genus of a long $\mathcal{N}=4$ representation. ${ }^{3}$ The observation of [1] was that the coefficients $A_{n}$ can be written in terms of dimensions of representations $H_{n}$ of the Mathieu group $\mathbb{M}_{24}$, so that

$$
\begin{aligned}
\phi_{\mathrm{K} 3}(\tau, z)= & \left(\operatorname{dim} H_{00}\right) \operatorname{ch}_{h=\frac{1}{4}, l=0}^{\mathcal{N}=4}(\tau, z)-\left(\operatorname{dim} H_{0}\right) \operatorname{ch}_{h=\frac{1}{4}, l=\frac{1}{2}}^{\mathcal{N}=4}(\tau, z) \\
& +\sum_{n=1}^{\infty}\left(\operatorname{dim} H_{n}\right) \operatorname{ch}_{h=n+\frac{1}{4}, l=\frac{1}{2}}^{\mathcal{N}=4}(\tau, z),
\end{aligned}
$$

where

$$
\begin{array}{rlrl}
H_{00} & =\mathbf{2 3}+\mathbf{1} & & H_{0}=2 \cdot \mathbf{1} \\
H_{1} & =\mathbf{4 5}+\overline{\mathbf{4 5}} & H_{2} & =\mathbf{2 3 1}+\overline{\mathbf{2 3 1}} \\
H_{3} & =\mathbf{7 7 0}+\overline{\mathbf{7 7 0}} & H_{4} & =\mathbf{2 2 7 7}+\overline{\mathbf{2 2 7 7}} \\
H_{5} & =2 \cdot \mathbf{5 7 9 6} & H_{6} & =2 \cdot \mathbf{3 5 2 0}+2 \cdot \mathbf{1 0 3 9 5} \\
H_{7} & =2 \cdot \mathbf{1 7 7 1}+2 \cdot \mathbf{2 0 2 4}+2 \cdot \mathbf{5 3 1 3}+2 \cdot \mathbf{5 7 9 6}+2 \cdot \mathbf{5 5 4 4}+2 \cdot \mathbf{1 0 3 9 5} .
\end{array}
$$

\footnotetext{
${ }^{3}$ Strictly speaking, the $\mathcal{N}=4$ representation with $n=0\left(h=\frac{1}{4}\right)$ is short, and thus $(2.7)$ for $h=\frac{1}{4}$ is not the elliptic genus of a single representation, but rather involves a sum of representations.
} 
The dimensions of the irreducible representations of $\mathbb{M}_{24}$ can be read off from the character table (see table 3). Note that we have absorbed the prefactor 2 in equation (1.11) of [1] into the definition of $A_{n}=\operatorname{dim}\left(H_{n}\right)$. Then we can write the $H_{n}$ in terms of real representations, so that, for example, $H_{1}$ is the sum of a pair of conjugate representations. The expression for $H_{7}$ was given in $[7,8]$ and differs from what was originally proposed in [1].

It is natural to conjecture that such a decomposition is the hallmark of a deeper structure underlying the elliptic genus of $\mathrm{K} 3$, see (1.2). In order to test this idea, the 'twining elliptic genera', i.e. the analogues of the McKay-Thompson series of Monstrous Moonshine, were considered in $[7,8]$. These twining genera are obtained from the elliptic genus upon inserting a group element $g \in \mathbb{M}_{24}$ into the trace

$$
\phi_{g}(\tau, z)=\frac{1}{2} \operatorname{Tr}_{\mathcal{H}_{\mathrm{RR}}}\left(g q^{L_{0}-\frac{c}{24}} e^{2 \pi i z J_{0}}(-1)^{F} \bar{q}^{\bar{L}_{0}-\frac{\bar{c}}{24}}(-1)^{\bar{F}}\right) .
$$

As in [8], we shall normalise the twining characters so that $\phi_{1 \mathrm{~A}}(\tau, z)=\frac{1}{2} \phi_{\mathrm{K} 3}(\tau, z)$ is directly equal to the standard weak Jacobi form $\phi_{0,1}$ (see appendix A). Technically speaking, $\phi_{g}$ is simply obtained from (2.8) by replacing the dimensions $A_{n}=\operatorname{dim}\left(H_{n}\right)$ by the trace of $g$ over $H_{n}, A_{n}(g)=\operatorname{Tr}_{H_{n}}(g)$, i.e.

$$
\begin{gathered}
\phi_{g}(\tau, z)=\frac{1}{2}\left[\operatorname{Tr}_{H_{00}}(g) \operatorname{ch}_{h=\frac{1}{4}, l=0}^{\mathcal{N}=4}(\tau, z)-\operatorname{Tr}_{H_{0}}(g) \operatorname{ch}_{h=\frac{1}{4}, l=\frac{1}{2}}^{\mathcal{N}=4}(\tau, z)\right. \\
\left.+\sum_{n=1}^{\infty} \operatorname{Tr}_{H_{n}}(g) \operatorname{ch}_{h=n+\frac{1}{4}, l=\frac{1}{2}}^{\mathcal{N}=4}(\tau, z)\right] .
\end{gathered}
$$

The character of $g$ only depends on its conjugacy class, and thus the various traces can be read off from the character table of $\mathbb{M}_{24}$, see table 3 .

As discussed in more detail in the next section, the twining genera are expected to be Jacobi forms under suitable congruence subgroups of $\operatorname{SL}(2, \mathbb{Z})$. This was confirmed for a number of conjugacy classes in $[7,8]$. In the next section we shall complete this programme by determining the twining characters for all remaining conjugacy classes. We shall furthermore show that they have the appropriate modular properties.

\section{Modular properties of the twining genera}

Using standard conformal field theory arguments, it was argued in $[7,8]$ that the twining genera $\phi_{g}$ should transform as Jacobi forms of index 1 and weight 0 , possibly up to a phase, under the congruence subgroup $\Gamma_{0}(N)$. More specifically, this means that $\phi_{g}$ satisfies $(2.3)$ for all $\ell, \ell^{\prime} \in \mathbb{Z}$, while (2.2) only holds for

$$
\left(\begin{array}{ll}
a & b \\
c & d
\end{array}\right) \in \Gamma_{0}(N)=\left\{\left(\begin{array}{ll}
a & b \\
c & d
\end{array}\right) \in \mathrm{SL}(2, \mathbb{Z}) \mid c \equiv 0 \bmod N\right\},
$$

where $N$ is the order of $g$. In [7], this was explicitly verified for the conjugacy classes ${ }^{4}$

$$
1 \mathrm{~A}, 2 \mathrm{~A}, 3 \mathrm{~A}, 4 \mathrm{~B}, 5 \mathrm{~A}, 6 \mathrm{~A}, 7 \mathrm{AB}, 8 \mathrm{~A}, 11 \mathrm{~A}, 14 \mathrm{AB}, 15 \mathrm{AB}, 23 \mathrm{AB} \text {. }
$$

\footnotetext{
${ }^{4}$ The classes which are power conjugated, for example $7 \mathrm{~A}$ and $7 \mathrm{~B}$, give rise to the same twining genus, so that we denote them as a unique class $7 \mathrm{AB}$. The twining genera for the classes $1 \mathrm{~A}, 2 \mathrm{~A}, 3 \mathrm{~A}, 4 \mathrm{~B}, 5 \mathrm{~A}$ and $6 \mathrm{~A}$ were also found in $[8]$.
} 
These classes are characterised by the condition $\phi_{g}(\tau, 0) \neq 0$. This is equivalent to the condition that a representative of the class is contained in the subgroup $\mathbb{M}_{23} \subset \mathbb{M}_{24}$, where we think of $\mathbb{M}_{24}$ as a subgroup of $S_{24}$, the permutation group of 24 points, and define $\mathbb{M}_{23} \subset \mathbb{M}_{24}$ to be the subgroup fixing, say, the first point. All geometric symmetries of K3 at a suitable point in moduli space lie in this subgroup [15, 16], and thus some of them can be calculated from first principles, see also [10]. For all of them the multiplier system turned out to be trivial [7]. The situation is more difficult for the remaining conjugacy classes

$$
2 \mathrm{~B}, 3 \mathrm{~B}, 4 \mathrm{~A}, 4 \mathrm{C}, 6 \mathrm{~B}, 10 \mathrm{~A}, 12 \mathrm{~A}, 12 \mathrm{~B}, 21 \mathrm{AB} \text {, }
$$

since there is no a priori method to determine them. In [8], explicit formulae were found for the first few of them by combining the constraints from modularity with the knowledge of the first few coefficients. ${ }^{5}$ In fact, the analysis of [8] was performed for the NS-sector version of the twining genus, the twining character

$$
\chi_{g}(\tau, z)=\exp \left[2 \pi i\left(\frac{\tau}{4}+z+\frac{1}{2}\right)\right] \phi_{g}\left(\tau, z+\frac{\tau}{2}+\frac{1}{2}\right)
$$

evaluated at $z=0$. The advantage of this approach is that one can work with standard modular functions (rather than Jacobi forms). The price one has to pay, on the other hand, is that part of the modular invariance is broken, and that multiplier phases are introduced for certain modular transformations. The latter property turned out to be a blessing in disguise since it suggested that multiplier phases may naturally appear in the modular transformations. Indeed, in [8] the twining characters were determined for all elements $g$ up to order $o(g) \leq 6$, and it was found that the classes in (3.2) and in (3.3) appear to behave very similarly. This suggests that also the twining genera $\phi_{g}$ associated to (3.3) should be invariant under $\Gamma_{0}(N)$, possibly up to non-trivial phases.

The appearance of a multiplier system in the transformation rule for the twining genera is certainly consistent with standard CFT arguments. In fact, this phenomenon also occurs for several McKay-Thompson series (and, more generally, for replicable functions [17]), which are the analogues of the twining characters in the context of Monstrous Moonshine [5]. Recall that each McKay-Thompson series $T_{g}$ is associated with a certain discrete group

$$
\Gamma_{0}(N \mid h)=\left\{\left(\begin{array}{cc}
a & b / h \\
N c & d
\end{array}\right) \in \mathrm{SL}(2, \mathbb{R}) \mid a, b, c, d \in \mathbb{Z}\right\},
$$

where $N$ is the order of the Monster class $g$, and $h$ is some integer such that $h \mid \operatorname{gcd}(N, 24)$. This group (sometimes with the inclusion of some Atkin-Lehner involutions of $\Gamma_{0}(N)$, see [5]) is the (restricted) eigengroup of $T_{g}$, i.e. the group under which $T_{g}$ is invariant up to $h$-th roots of unity. In particular, $T_{g}$ is invariant (without any phases) under $\Gamma_{0}(N h) \subset$ $\Gamma_{0}(N)$, while under the cosets of $\Gamma_{0}(N \mid h) / \Gamma_{0}(N h)$, that are represented by

$$
\left(\begin{array}{cc}
1 & 1 / h \\
0 & 1
\end{array}\right) \quad \text { and } \quad\left(\begin{array}{cc}
1 & 0 \\
N & 1
\end{array}\right),
$$

\footnotetext{
${ }^{5}$ The twining characters for $2 \mathrm{~B}$ and $4 \mathrm{~A}$ were also found in [7].
} 


\begin{tabular}{|c|ccccccccc|}
\hline Class & $2 \mathrm{~B}$ & $3 \mathrm{~B}$ & $4 \mathrm{~A}$ & $4 \mathrm{C}$ & $6 \mathrm{~B}$ & $10 \mathrm{~A}$ & $12 \mathrm{~A}$ & $12 \mathrm{~B}$ & $21 \mathrm{AB}$ \\
\hline$h$ & 2 & 3 & 2 & 4 & 6 & 2 & 2 & 12 & 3 \\
\hline
\end{tabular}

Table 1. Value of $h$ for the conjugacy classes in (3.3).

it transforms as

$$
T_{g}\left(\tau+\frac{1}{h}\right)=e^{-\frac{2 \pi i}{h}} T_{g}(\tau) \quad \text { and } \quad T_{g}\left(\frac{\tau}{N \tau+1}\right)=e^{ \pm \frac{2 \pi i}{h}} T_{g}(\tau) .
$$

The two cosets in (3.6) generate $\Gamma_{0}(N \mid h)$, so that (3.7) uniquely determines the multiplier system under $\Gamma_{0}(N \mid h)$.

It is then natural to expect that analogous properties hold for the twining genera of $\mathbb{M}_{24}$. The most obvious generalisation would be to require the twining genus $\phi_{g}$, with $g \in \mathbb{M}_{24}$ and $o(g)=N$, to be a Jacobi form (with a suitable multiplier system) of weight 0 and index 1 under $\Gamma_{0}(N \mid h)$, for some $h \mid \operatorname{gcd}(N, 24)$. However, there is one immediate problem with this proposal: for $h>1, \Gamma_{0}(N \mid h)$ is not contained in $\operatorname{SL}(2, \mathbb{Z})$, and it is not clear how to define the action of the whole $\Gamma_{0}(N \mid h)$ on Jacobi forms. ${ }^{6}$ Thus we can only analyse the modular properties under the subgroup $\Gamma_{0}(N \mid h) \cap \mathrm{SL}(2, \mathbb{Z}) \cong \Gamma_{0}(N)$. This then leads to the following conjecture:

Conjecture. For all the conjugacy classes $g$ of $\mathbb{M}_{24}$, the twining character $\phi_{g}(\tau, z)$ is a Jacobi form of index one and weight zero under $\Gamma_{0}(N)$, with a multiplier system defined by

$$
\phi_{g}\left(\frac{a \tau+b}{c \tau+d}, \frac{z}{c \tau+d}\right)=e^{\frac{2 \pi i c}{N h}} e^{\frac{2 \pi i c z^{2}}{c \tau+d}} \phi_{g}(\tau, z), \quad\left(\begin{array}{ll}
a & b \\
c & d
\end{array}\right) \in \Gamma_{0}(N),
$$

where $N$ is the order of $g$ and $h \mid \operatorname{gcd}(N, 12)$. The multiplier system is trivial $(h=1)$ if and only if $g$ contains a representative in $\mathbb{M}_{23} \subset \mathbb{M}_{24}$.

For the classes in (3.2) that have representatives in $\mathbb{M}_{23}$ the conjecture has been shown in [7]. In the next subsections, we will show that the conjecture is also true for the remaining classes, i.e. the elements in (3.3), with the values of $h$ as given in table 1. Because of these non-trivial multiplier systems, the analysis is quite difficult, and we have applied two different strategies. First we have refined the method of [8] by considering the twining genus $\phi_{g}(\tau, z)$ as a function of $\tau$ at special values of $z$. These values are chosen in such a way that the $z$-dependent exponential factor in the transformation formula (3.8) cancels (part of) the $h$-th root of unity. If the phase is completely removed (as is the case for all but two classes), the resulting function is a modular function for $\Gamma_{0}(N)$, which can be easily analysed. Using this approach (as well as some guess work for the other two cases) we have succeeded in finding closed formulae for all the characters with $h>1$, see section 3.1.

The other strategy follows the idea advocated in [7] and consists of expanding the twining genus in terms of standard weak Jacobi forms. This reduces the problem to finding a

\footnotetext{
${ }^{6} \mathrm{~A}$ well-defined action of $\mathrm{SL}(2, \mathbb{R})$ on Jacobi forms can be defined, see [11]. However, this action does not respect the periodicity condition on $z$, and thus does not seem to be relevant in the current context.
} 
suitable modular form of weight two. This problem can be studied systematically, but usually leads to more complicated computations. In section 3.2 we shall explain the salient features of this analysis, while the explicit formulae for all characters are given in appendix C.

\subsection{Characters evaluated at fixed values of $z$}

Let $\phi(\tau, z)$ be a weak Jacobi form of weight 0 and index 1 , transforming as in (3.8), for some $N$ and $h$ with $h \mid \operatorname{gcd}(N, 12)$. Then, for any $k \in \mathbb{Z}$, we have

$$
\begin{aligned}
\phi\left(\frac{a \tau+b}{c \tau+d}, \frac{k}{h}\right) & =\phi\left(\frac{a \tau+b}{c \tau+d}, \frac{k(c \tau+d)}{h(c \tau+d)}\right)=e^{\frac{2 \pi i c}{N h}} e^{\frac{2 \pi i k^{2} c(c \tau+d)}{h^{2}}} \phi\left(\tau, \frac{k(c \tau+d)}{h}\right) \\
& =e^{\frac{2 \pi i c}{N h}} e^{\frac{2 \pi i k^{2} c(c \tau+d)}{h^{2}}} e^{-2 \pi i\left(\frac{k^{2} c^{2}}{h^{2}} \tau+2 \frac{k^{2} c d}{h^{2}}\right)} \phi\left(\tau, \frac{k d}{h}\right) \\
& =e^{2 \pi i c\left(-\frac{k^{2} d}{h^{2}}+\frac{1}{N h}\right)} \phi\left(\tau, \frac{k d}{h}\right), \quad \text { for } \quad\left(\begin{array}{ll}
a & b \\
c & d
\end{array}\right) \in \Gamma_{0}(N),
\end{aligned}
$$

where we have used (2.3) in the second line. (Note that $c \in N \mathbb{Z}$, and hence $\frac{c}{h} \in \mathbb{Z}$.) Let us define

$$
\Phi^{(h)}(\tau)=\frac{1}{\varphi(h)} \sum_{k \in(\mathbb{Z} / h \mathbb{Z})^{*}} \phi\left(\tau, \frac{k}{h}\right),
$$

where $(\mathbb{Z} / h \mathbb{Z})^{*}$ is the set of totatives of $h$, i.e. the positive integers smaller than $h$ that are relatively prime to $h$, and the Euler totient function $\varphi(h)$ is the number of all such totatives. This definition simplifies considerably in concrete examples

$$
\begin{aligned}
\Phi^{(h)}(\tau) & =\phi\left(\tau, \frac{1}{h}\right), & h=2,3,4,6, \\
\Phi^{(12)}(\tau) & =\frac{1}{2}\left(\phi\left(\tau, \frac{1}{12}\right)+\phi\left(\tau, \frac{5}{12}\right)\right), &
\end{aligned}
$$

because $\phi(\tau, z)=\phi(\tau,-z)$ for Jacobi forms of even weight. It is easy to verify that, for all $h \mid 12$, the condition $\operatorname{gcd}(k, h)=1$ implies $k^{2} \equiv 1 \bmod h$. Furthermore, for $\left(\begin{array}{ll}a & b \\ c & d\end{array}\right) \in \Gamma_{0}(N)$, the condition $a d-b c=1$ implies $\operatorname{gcd}(d, h)=1$, so that the map $k \mapsto k d$ is bijective on $(\mathbb{Z} / h \mathbb{Z})^{*}$. Thus we conclude

$$
\Phi^{(h)}\left(\frac{a \tau+b}{c \tau+d}\right)=e^{2 \pi i c\left(-\frac{d}{h^{2}}+\frac{1}{N h}\right)} \Phi^{(h)}(\tau), \quad\left(\begin{array}{ll}
a & b \\
c & d
\end{array}\right) \in \Gamma_{0}(N),
$$

and it follows immediately that $\Phi^{(h)}(\tau)$ is invariant under $\Gamma_{0}(N h)$. Since $h$ divides $N$, the cosets of $\Gamma_{0}(N) / \Gamma_{0}(N h)$ are generated by $\left(\begin{array}{ll}1 & 0 \\ N & 1\end{array}\right)$, and the phase in (3.13) cancels under this transformation if and only if

$$
\frac{N}{h} \equiv 1 \bmod h
$$

Thus $\Phi^{(h)}$ is actually invariant under $\Gamma_{0}(N)$ if (3.14) holds. In this case, it is easy to obtain a closed formula for $\Phi_{g}^{(h)}$. In fact, with the exception of $N=21$, all the groups $\Gamma_{0}(N)$ we 
are interested in are genus zero, ${ }^{7}$ so that all modular functions must be rational functions of the corresponding Hauptmodul.

As it turns out the condition (3.14) is satisfied for all classes in (3.3), with the exception of $4 \mathrm{~A}$ and $12 \mathrm{~A}$ for which $h=2$ and hence $N / h \equiv 0 \bmod h$. In all other cases we found a modular function for $\Gamma_{0}(N)$ which matches the first few coefficients in the $q$-expansion that can be determined from (2.9) and (2.10). This function is either a constant or a fractional linear transformation of the Hauptmodul (in fact, a McKay-Thompson series) for $\Gamma_{0}(N)$. For the class $21 \mathrm{AB}$ it is a rational function in the McKay-Thompson series $T_{[21 \mathrm{~B}]}$ and $T_{[21 \mathrm{D}]}$, that are modular functions for $\Gamma_{0}(21)$ (for a $q$-expansion of these McKay Thompson series see appendix A). Our explicit expressions are:

$$
\begin{array}{rlrl}
\Phi_{2 \mathrm{~B}}^{(2)}(\tau) & =-4 & h & =2 \\
\Phi_{3 \mathrm{~B}}^{(3)}(\tau) & =-3 & h & =3 \\
\Phi_{4 \mathrm{C}}^{(4)}(\tau) & =-2 & h & =4 \\
\Phi_{6 \mathrm{~B}}^{(6)}(\tau) & =-1 & h & =6 \\
\Phi_{10 \mathrm{~A}}^{(2)}(\tau) & =-4 \frac{\eta(5 \tau) \eta(2 \tau)^{5}}{\eta(10 \tau) \eta(\tau)^{5}}=\frac{-20}{T_{[10 \mathrm{E}]}-3}-4 & h & =2 \\
\Phi_{12 \mathrm{~B}}^{(12)}(\tau) & =-2-6 \frac{\eta(2 \tau)^{2} \eta(3 \tau) \eta(12 \tau)^{3}}{\eta(\tau)^{3} \eta(4 \tau) \eta(6 \tau)^{2}}=\frac{-6}{T_{[12 \mathrm{I}]}-3}-2 & h & =12 \\
\Phi_{21 A B}^{(3)}(\tau) & =\frac{1}{2}-\frac{7}{2} \frac{\eta(3 \tau) \eta(7 \tau)^{3}}{\eta(\tau)^{3} \eta(21 \tau)}=\frac{-T_{[21 \mathrm{~B}]}+7 T_{[21 \mathrm{D}]}+15}{-2 T_{[21 \mathrm{~B}]}+2} & h & =3 .
\end{array}
$$

Since $\phi_{g}(\tau, z)$ is a Jacobi form of index one, we have

$$
\phi_{g}(\tau, z)=\phi_{g}(\tau, 0) \frac{\vartheta_{2}(\tau, z)^{2}}{\vartheta_{2}(\tau, 0)^{2}}+\phi_{g}\left(\tau, \frac{1}{2}\right) \frac{\vartheta_{1}(\tau, z)^{2}}{\vartheta_{2}(\tau, 0)^{2}}
$$

so that (3.15) immediately gives a formula for the corresponding character at generic $z$

$$
\phi_{g}(\tau, z)=\Phi_{g}^{(h)}(\tau) \varphi(h)\left(\sum_{k \in(\mathbb{Z} / h \mathbb{Z})^{*}} \vartheta_{1}\left(\tau, \frac{k}{h}\right)^{2}\right)^{-1} \vartheta_{1}(\tau, z)^{2},
$$

where we also used the fact that, for all $g$ in (3.3),

$$
\phi_{g}(\tau, 0)=\frac{1}{2} \operatorname{Tr}_{\mathbf{2 3} \oplus \mathbf{1}}(g)=0 .
$$

The remaining two cases, $4 \mathrm{~A}$ and $12 \mathrm{~A}$, are not invariant under $\Gamma_{0}(N)$, and thus require more work. For $4 \mathrm{~A}$ a closed formula was already found in $[7,8]$

$$
\phi_{4 \mathrm{~A}}\left(\tau, \frac{1}{2}\right)=-4-32 \frac{\eta(2 \tau)^{2} \eta(8 \tau)^{4}}{\eta(\tau)^{4} \eta(4 \tau)^{2}}=-4-\frac{32}{T_{8 \mathrm{E}}(\tau)+4} \quad h=2,
$$

\footnotetext{
${ }^{7}$ Genus zero here means that the Riemann surface obtained by quotienting the upper half-plane $\mathbb{H}$ by $\Gamma_{0}(N)$ has the topology of the sphere.
} 
and it is easy to verify that the corresponding $\phi_{4 \mathrm{~A}}(\tau, z)$ transforms as in (3.8) under $\Gamma_{0}(4)$ with $h=2$. We have also managed to find a closed formula for the NS-character $\chi_{g}$ of $12 \mathrm{~A}$ at $z=\frac{1}{6}$

$$
\chi_{12 \mathrm{~A}}\left(\tau, \frac{1}{6}\right)=\frac{\eta(\tau) \eta\left(\frac{3 \tau}{2}\right)^{2} \eta(4 \tau)^{2} \eta(6 \tau)^{3}}{\eta(2 \tau) \eta\left(\frac{\tau}{2}\right)^{2} \eta(12 \tau)^{2} \eta(3 \tau)^{3}},
$$

from which the function $\phi_{12 \mathrm{~A}}(\tau, z)$ can be reconstructed. However, it is easier to analyse the modular properties of $\phi_{12 \mathrm{~A}}(\tau, z)$ using the methods described in the next subsection.

\subsection{Studying the modular forms of weight two}

Every weak Jacobi form of index 1 can be written as a linear combination of the standard Jacobi forms $\phi_{0,1}$ and $\phi_{-2,1}$ of weight 0 and -2 , respectively (see appendix A), where the coefficients lie in the space of modular forms under the relevant subgroup of $\operatorname{SL}(2, \mathbb{Z})[11]$. In particular,

$$
\phi_{g}(\tau, z)=B_{g} \phi_{0,1}(\tau, z)+F_{g}(\tau) \phi_{-2,1}(\tau, z),
$$

where $B_{g}$ is the constant

$$
B_{g}=\frac{1}{12} \phi_{g}(\tau, 0)=\frac{1}{24} \operatorname{Tr}_{\mathbf{2 3} \oplus \mathbf{1}}(g),
$$

while $F_{g}$ is a suitable modular form of weight two. If $g$ is in (3.2), the phases in (3.8) are trivial, and $F_{g} \in M_{2}\left(\Gamma_{0}(N)\right)$, where $M_{k}(\Gamma)$ denotes the space of modular form of weight $k$ under the group $\Gamma$. In [7], it has been shown that, for all $g$ in (3.2), there is a unique $F_{g} \in$ $M_{2}\left(\Gamma_{0}(N)\right)$ matching the known coefficients of the $q$-expansion of $\phi_{g}$ (see also appendix $\mathrm{C}$ ).

If $g$ is one of the classes in (3.3), then $B_{g}=0$ and the conjecture (3.8) would imply that $F_{g}$ transforms as

$$
F_{g}\left(\frac{a \tau+b}{c \tau+d}\right)=e^{\frac{2 \pi i c}{N h}}(c \tau+d)^{2} F(\tau), \quad\left(\begin{array}{ll}
a & b \\
c & d
\end{array}\right) \in \Gamma_{0}(N),
$$

where $N$ is the order of $g$ and $h \mid \operatorname{gcd}(N, 12)$. In particular, this means that

$$
F_{g} \in M_{2}\left(\Gamma_{0}(N h)\right), \quad F_{g}^{h} \in M_{2 h}\left(\Gamma_{0}(N)\right) .
$$

Conversely, if these conditions hold, then $F_{g}$ transforms as a modular form of weight 2 under $\Gamma_{0}(N)$ up to a certain $h$-th root of unity $e^{\frac{2 \pi i r c}{N h}}$, for some $r \in \mathbb{Z}$.

For all $g$ in (3.3), we have found modular forms $F_{g} \in M_{2}\left(\Gamma_{0}(N h)\right)$ for the above values of $h$ that reproduce the first few coefficients of $q$ as determined from (2.9) and (2.10), and satisfy $F_{g}^{h} \in M_{2 h}\left(\Gamma_{0}(N)\right)$. The explicit expressions for $F_{g}$ (and $F_{g}^{h}$ ) in terms of suitable bases of $M_{2}\left(\Gamma_{0}(N h)\right)$ (and $\left.M_{2 h}\left(\Gamma_{0}(N)\right)\right)$ are quite complicated, and are therefore only given in appendix $\mathrm{C}$.

It remains to prove that the $h$-th root of unity $e^{\frac{2 \pi i r c}{N h}}$ in the modular transformation of $F_{g}$ is the same as in (3.8), i.e. that $r=1$. We are mainly interested in the class $12 \mathrm{~A}$ because the other cases have already been dealt with in the previous subsection. For $12 \mathrm{~A}$ we have found $h=2$, and since the phase is non-trivial (as can be easily checked), the only possibility is $r=1$. This completes our analysis. 


\section{Decomposition into irreducible representations}

In the previous sections, we provided explicit expressions for all twining genera, matching the first few coefficients in (2.12) and transforming as in (3.8). The knowledge of all such characters also leads to another very stringent consistency check of the proposal. It allows us to check whether there are indeed underlying $\mathbb{M}_{24}$ representations $H_{n}$ such that $A_{n}(g)=\operatorname{Tr}_{H_{n}}(g)$, where $A_{n}(g)$ is the coefficient replacing $A_{n}$ in $\phi_{g}$, see (2.12).

In order to understand how this works, let us assume that $A_{n}(g)=\operatorname{Tr}_{H_{n}}(g)$, where $H_{n}$ is a $\mathbb{M}_{24}$ representation which we decompose as

$$
H_{n}=\bigoplus_{i=1}^{26} h_{n, i} R_{i}
$$

where $i=1, \ldots, 26$ labels the different irreducible representations of $\mathbb{M}_{24}$, numbered as in table 3 . To start with we rewrite $(2.12)$ as

$$
-\operatorname{Tr}_{H_{0}}(g)+\sum_{n=1}^{\infty} \operatorname{Tr}_{H_{n}}(g) q^{n}=q^{\frac{1}{8}} \frac{\eta(\tau)^{3}}{\vartheta_{1}(\tau, z)^{2}}\left(2 \phi_{g}(\tau, z)-\operatorname{Tr}_{H_{00}}(g) \operatorname{ch}_{h=\frac{1}{4}, l=0}^{\mathcal{N}=4}(\tau, z)\right),
$$

where we have used (2.7). Next we recall that the characters of a finite group satisfy the orthonormality relations

$$
\sum_{g} c(g) \overline{\operatorname{Tr}_{R}(g)} \operatorname{Tr}_{R^{\prime}}(g)= \begin{cases}1 & \text { if } R \cong R^{\prime} \\ 0 & \text { otherwise }\end{cases}
$$

where $R$ and $R^{\prime}$ are two irreducible representations of $\mathbb{M}_{24}$. Here the sum runs over all conjugacy classes of $\mathbb{M}_{24}$, and $c(g)^{-1}$ is the order of the centraliser of $g$

$$
c(g)=\frac{n(g)}{\left|\mathbb{M}_{24}\right|}, \quad \text { with } \quad\left|\mathbb{M}_{24}\right|=2^{10} \cdot 3^{3} \cdot 5 \cdot 7 \cdot 11 \cdot 23
$$

the order of $\mathbb{M}_{24}$ and $n(g)$ the number of elements in the conjugacy class of $g$. Using (4.3) we now obtain from $(4.2)$

$$
-2 \delta_{i, 1}+\sum_{n=1}^{\infty} h_{n, i} q^{n}=q^{\frac{1}{8}} \frac{\eta(\tau)^{3}}{\vartheta_{1}(\tau, z)^{2}}\left(2 \sum_{g} c(g) \overline{\operatorname{Tr}_{R_{i}}(g)} \phi_{g}(\tau, z)-\left(\delta_{i, 1}+\delta_{i, 2}\right) \operatorname{ch}_{h=\frac{1}{4}, l=0}^{\mathcal{N}=4}(\tau, z)\right),
$$

where $i=1, \ldots, 26$. In deriving (4.5) we have used that $H_{00}=R_{1} \oplus R_{2}=\mathbf{1} \oplus \mathbf{2 3}$ and $H_{0}=2 \cdot R_{1}=2 \cdot \mathbf{1}$. For $i>2,(4.5)$ simplifies, and we obtain

$$
\sum_{n=1}^{\infty} h_{n, i} q^{n}=-2 q^{\frac{1}{8}} \frac{\sum_{g} c(g) \overline{\operatorname{Tr}_{R_{i}}(g)} F_{g}(\tau)}{\eta(\tau)^{3}}=-2 \frac{\sum_{g} c(g) \overline{\operatorname{Tr}_{R_{i}}(g)} F_{g}(\tau)}{\prod_{n=1}^{\infty}\left(1-q^{n}\right)^{3}}, \quad i>2,
$$

where we used (3.21), as well as (3.22) and (4.3) again. Furthermore, we have plugged in the explicit expression for $\phi_{-2,1}$ from (A.7).

The character values $\operatorname{Tr}_{R_{i}}(g)$ are given in table 3 , and the explicit values of $c(g)$ are tabulated in table 2 . The analysis of the previous sections provide closed formulae for the 


\begin{tabular}{|c|c||c|c||c|c|}
\hline Class & $c(g)^{-1}$ & Class & $c(g)^{-1}$ & Class & $c(g)^{-1}$ \\
\hline $1 \mathrm{~A}$ & 244823040 & $6 \mathrm{~A}$ & 24 & $4 \mathrm{C}$ & 96 \\
$2 \mathrm{~A}$ & 21504 & $11 \mathrm{~A}$ & 11 & $3 \mathrm{~B}$ & 504 \\
$3 \mathrm{~A}$ & 1080 & $15 \mathrm{AB}$ & 15 & $2 \mathrm{~B}$ & 7680 \\
$5 \mathrm{~A}$ & 60 & $14 \mathrm{AB}$ & 14 & $10 \mathrm{~A}$ & 20 \\
$4 \mathrm{~B}$ & 128 & $23 \mathrm{AB}$ & 23 & $21 \mathrm{AB}$ & 21 \\
$7 \mathrm{AB}$ & 42 & $12 \mathrm{~B}$ & 12 & $4 \mathrm{~A}$ & 384 \\
$8 \mathrm{~A}$ & 16 & $6 \mathrm{~B}$ & 24 & $12 \mathrm{~A}$ & 12 \\
\hline
\end{tabular}

Table 2. Order $c(g)^{-1}$ of the centraliser of the class $g$.

twining characters $\phi_{g}(\tau, z)$ for all conjugacy classes $g$ in $\mathbb{M}_{24}$, so that the right hand side of (4.5) can be easily evaluated. Thus we can determine the multiplicities $h_{n, i}$ explicitly. The statement that there are underlying $\mathbb{M}_{24}$ representations $H_{n}$ is now simply equivalent to the property of the multiplicities $h_{n, i}$ to be non-negative integers. We have worked out these multiplicities for $n \leq 500$, and all of them are indeed non-negative integers; the explicit values for $n \leq 30$ are listed in table 4 .

Since all the characters we have constructed have real coefficients, the multiplicities of conjugate representations are always equal, so that all $H_{n}$ are real representations, as expected. It is also remarkable that the multiplicities of the real irreducible representations in (4.1) are always even, at least, up to $n=500$ (see also [7]). This suggests that the actual symmetry group may be slightly bigger than the Mathieu group $\mathbb{M}_{24}$.

\section{Conclusions}

In this paper we have accumulated compelling evidence for the conjecture of Eguchi, Ooguri and Tachikawa [1] that the states contributing to the elliptic genus of K3 carry an action of the Mathieu group $\mathbb{M}_{24}$. More specifically, we have found closed form expressions for the twining genera of $\mathrm{K} 3$ for all conjugacy classes of $\mathbb{M}_{24}$, thus completing the programme initiated in $[7,8]$. We have shown that the twining genera transform indeed as Jacobi forms of index one and weight zero under $\Gamma_{0}(N)$, where $N$ is the order of the corresponding group element. The twining genera of the conjugacy classes that have no representative contained in $\mathbb{M}_{23} \subset \mathbb{M}_{24}$ have a non-trivial multiplier system, which we have identified, see eq. (3.8).

The explicit knowledge of all twining genera allows one to determine the decomposition of the elliptic genus of $\mathrm{K} 3$ in terms of $\mathbb{M}_{24}$ representations, and we have checked that the multiplicities with which these representations appear are indeed non-negative integers, at least for the first 500 coefficients - see also table 4 for explicit results for $n \leq 30$. This is a highly non-trivial consistency check; indeed, if we were able to show that all of these multiplicities are non-negative integers, this would effectively prove the conjecture of [1]. 


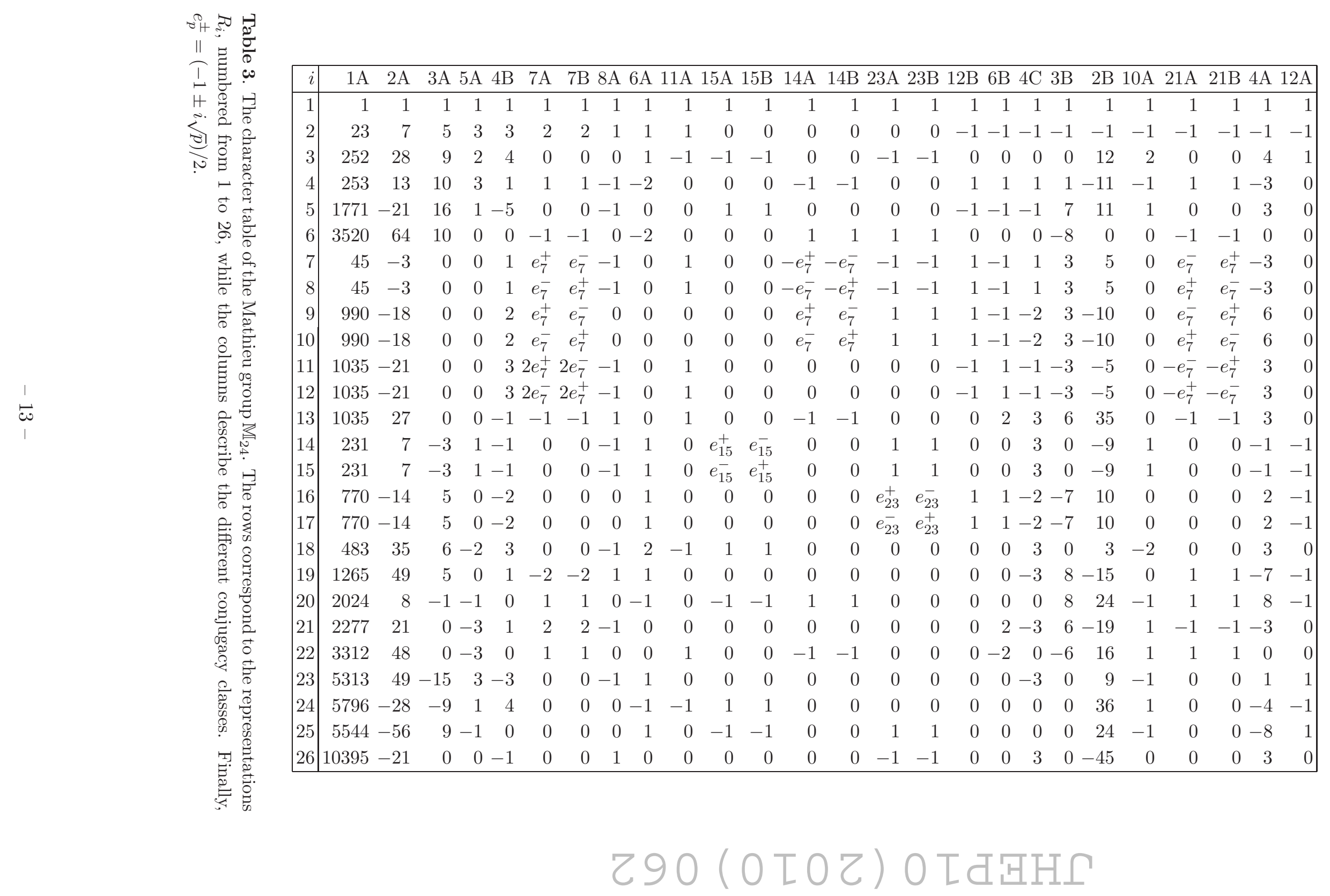




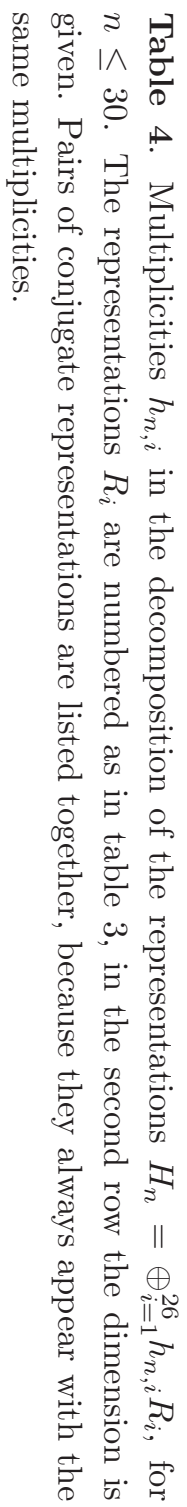

\begin{tabular}{|c|c|c|c|c|c|c|c|c|c|c|c|c|c|c|c|c|c|c|c|c|c|}
\hline \begin{tabular}{|l|}
$i$ \\
\end{tabular} & 1 & 2 & 3 & 4 & 5 & 6 & 7,8 & 9,10 & 11,12 & 13 & 14,15 & 16,17 & 18 & 19 & 20 & 21 & 22 & 23 & 24 & 25 & 26 \\
\hline \begin{tabular}{|l|}
$n$ \\
\end{tabular} & 1 & 23 & 252 & 253 & 1771 & 3520 & 45 & 990 & 1035 & 1035 & 231 & 770 & 483 & 1265 & 2024 & 2277 & 3312 & 5313 & 5796 & 5544 & 10395 \\
\hline 1 & 0 & 0 & 0 & 0 & 0 & 0 & 1 & 0 & 0 & 0 & 0 & 0 & 0 & 0 & 0 & 0 & 0 & 0 & 0 & 0 & 0 \\
\hline 2 & 0 & 0 & 0 & 0 & 0 & 0 & 0 & 0 & 0 & 0 & 1 & 0 & 0 & 0 & 0 & 0 & 0 & 0 & 0 & 0 & 0 \\
\hline 3 & 0 & 0 & 0 & 0 & 0 & 0 & 0 & 0 & 0 & 0 & 0 & 1 & 0 & 0 & 0 & 0 & 0 & 0 & 0 & 0 & 0 \\
\hline 4 & 0 & 0 & 0 & 0 & 0 & 0 & 0 & 0 & 0 & 0 & 0 & 0 & 0 & 0 & 0 & 2 & 0 & 0 & 0 & 0 & 0 \\
\hline 5 & 0 & 0 & 0 & 0 & 0 & 0 & 0 & 0 & 0 & 0 & 0 & 0 & 0 & 0 & 0 & 0 & 0 & 0 & 2 & 0 & 0 \\
\hline 6 & 0 & 0 & 0 & 0 & 0 & 2 & 0 & 0 & 0 & 0 & 0 & 0 & 0 & 0 & 0 & 0 & 0 & 0 & 0 & 0 & 2 \\
\hline 7 & 0 & 0 & 0 & 0 & 2 & 0 & 0 & 0 & 0 & 0 & 0 & 0 & 0 & 0 & 2 & 0 & 0 & 2 & 2 & 2 & 2 \\
\hline 8 & 0 & 0 & 0 & 0 & 0 & 2 & 0 & 1 & 1 & 0 & 0 & 0 & 0 & 2 & 0 & 2 & 2 & 4 & 2 & 2 & 6 \\
\hline 9 & 0 & 0 & 0 & 0 & 2 & 4 & 0 & 0 & 2 & 2 & 0 & 2 & 2 & 0 & 2 & 2 & 4 & 4 & 8 & 8 & 10 \\
\hline 10 & 0 & 0 & 0 & 2 & 4 & 8 & 0 & 2 & 2 & 2 & 2 & 0 & 2 & 4 & 4 & 6 & 6 & 12 & 10 & 10 & 24 \\
\hline 11 & 0 & 0 & 0 & 0 & 8 & 12 & 0 & 4 & 4 & 6 & 0 & 4 & 0 & 2 & 10 & 8 & 14 & 22 & 26 & 24 & 40 \\
\hline 12 & 0 & 2 & 2 & 4 & 12 & 30 & 0 & 8 & 8 & 4 & 2 & 6 & 4 & 12 & 12 & 18 & 26 & 40 & 40 & 38 & 80 \\
\hline 13 & 0 & 0 & 4 & 2 & 26 & 44 & 2 & 14 & 14 & 18 & 2 & 10 & 6 & 16 & 30 & 28 & 44 & 70 & 84 & 80 & 136 \\
\hline 14 & 0 & 0 & 4 & 6 & 38 & 86 & 0 & 24 & 24 & 22 & 8 & 16 & 14 & 34 & 46 & 58 & 80 & 128 & 132 & 126 & 254 \\
\hline 15 & 0 & 0 & 12 & 8 & 78 & 144 & 2 & 40 & 44 & 46 & 8 & 38 & 18 & 46 & 86 & 88 & 138 & 218 & 246 & 238 & 424 \\
\hline 16 & 0 & 2 & 18 & 22 & 122 & 252 & 2 & 72 & 72 & 68 & 18 & 50 & 36 & 100 & 140 & 170 & 232 & 378 & 400 & 382 & 742 \\
\hline 17 & 0 & 2 & 30 & 26 & 212 & 410 & 8 & 116 & 124 & 130 & 25 & 94 & 54 & 140 & 246 & 262 & 392 & 630 & 704 & 670 & 1222 \\
\hline 18 & 0 & 6 & 50 & 58 & 342 & 704 & 6 & 194 & 202 & 192 & 50 & 148 & 100 & 256 & 388 & 454 & 654 & 1044 & 1120 & 1074 & 2058 \\
\hline 19 & 0 & 4 & 80 & 72 & 582 & 1116 & 18 & 318 & 332 & 346 & 68 & 252 & 150 & 394 & 664 & 722 & 1062 & 1702 & 1880 & 1800 & 3320 \\
\hline 20 & 0 & 14 & 128 & 138 & 904 & 1836 & 20 & 516 & 536 & 520 & 126 & 390 & 254 & 676 & 1036 & 1196 & 1716 & 2764 & 2980 & 2846 & 5408 \\
\hline 21 & 2 & 20 & 214 & 200 & 1476 & 2902 & 40 & 814 & 860 & 872 & 182 & 652 & 396 & 1020 & 1684 & 1862 & 2742 & 4384 & 4828 & 4622 & 8572 \\
\hline 22 & 2 & 32 & 328 & 346 & 2302 & 4616 & 55 & 1298 & 1348 & 1336 & 314 & 988 & 640 & 1686 & 2630 & 3000 & 4324 & 6950 & 7532 & 7204 & 13620 \\
\hline 23 & 2 & 40 & 512 & 496 & 3638 & 7166 & 98 & 2020 & 2118 & 2144 & 460 & 1590 & 972 & 2546 & 4162 & 4624 & 6768 & 10856 & 11898 & 11376 & 21204 \\
\hline 24 & 0 & 80 & 798 & 824 & 5584 & 11192 & 132 & 3140 & 3278 & 3236 & 744 & 2426 & 1544 & 4050 & 6376 & 7248 & 10500 & 16834 & 18294 & 17504 & 32976 \\
\hline 25 & 8 & 108 & 1232 & 1208 & 8654 & 17084 & 234 & 4814 & 5038 & 5084 & 1106 & 3764 & 2336 & 6108 & 9892 & 11042 & 16112 & 25840 & 28288 & 27056 & 50524 \\
\hline 26 & 6 & 174 & 1860 & 1904 & 13090 & 26148 & 322 & 7348 & 7670 & 7626 & 1742 & 5677 & 3602 & 9444 & 14968 & 16940 & 24566 & 39428 & 42894 & 41022 & 77176 \\
\hline 27 & 12 & 252 & 2836 & 2802 & 19914 & 39436 & 514 & 11092 & 11618 & 11666 & 2560 & 8688 & 5394 & 14100 & 22744 & 25462 & 37148 & 59564 & 65114 & 62294 & 116494 \\
\hline 28 & 16 & 398 & 4238 & 4310 & 29772 & 59330 & 742 & 16686 & 17418 & 17356 & 3922 & 12912 & 8160 & 21414 & $=34026$ & 38434 & 55764 & 89490 & 97456 & 93218 & 175146 \\
\hline 29 & 26 & 560 & 6328 & 6286 & 44512 & 88280 & 1154 & 24840 & 25994 & 26078 & 5758 & 19380 & 12090 & 31636 & 50892 & 57068 & 83146 & 133356 & 145690 & 139342 & 260828 \\
\hline 30 & 34 & 876 & 9368 & 9486 & 65776 & 131020 & 1642 & 36824 & 38480 & 38368 & 8642 & 28580 & 18008 & 47172 & 75158 & 84776 & 123176 & 197596 & 215318 & 205970 & 386724 \\
\hline
\end{tabular}

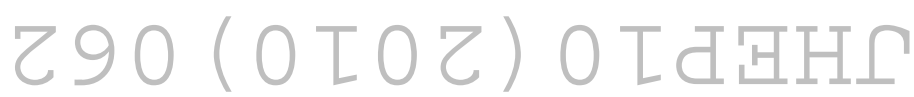


Another, more conceptual, proof of the conjecture would consist of constructing explicitly the action of $\mathbb{M}_{24}$ on the BPS states contributing to the elliptic genus of K3. For some generators in $\mathbb{M}_{23} \subset \mathbb{M}_{24}$ this can be done fairly directly since they describe geometric automorphisms of the K3 surface [15, 16], see also [18] for some recent progress in this direction. However, it seems unlikely that these purely geometrical symmetries will suffice to account for the full $\mathbb{M}_{24}$ symmetry of the elliptic genus. In fact, one may guess that one will need to consider the full moduli space of the non-linear sigma model in order to achieve this. Thus at least some of the required symmetries may have an interpretation in terms of truly stringy symmetries, such as e.g. T-duality. It would be very interesting to explore these ideas in more detail [19].

On a more technical note, it is amusing to note (see also [8]) that, in contrast to the McKay-Thompson series in Monstrous Moonshine [5], not all twining genera seem to be Hauptmoduls for genus zero congruence subgroups (although many are). For example, the twining character $21 \mathrm{AB}$, for which the relevant modular group, $\Gamma_{0}(21)$, is not genus 0 . This fact is also reflected in equation (3.15) where we have given the explicit expression of $\Phi_{21 \mathrm{AB}}^{(3)}(\tau)$ as a modular form of $\Gamma_{0}(21)$. Unlike the remaining twining characters, it cannot be written as a rational function of a single modular function (the Hauptmodul) but rather involves two functions (the McKay-Thompson series $T_{[21 \mathrm{~B}]}$ and $T_{[21 \mathrm{D}]}$ ). It would be very interesting to understand what the significance of the genus zero property in this context is.

\section{Acknowledgments}

We thank Terry Gannon for useful communications and John McKay for inspiring discussions. The research of MRG and SH is partially supported by a grant from the Swiss National Science Foundation, and the research of RV is supported by an INFN Fellowship.

\section{A Definitions}

Our conventions for the Dedekind eta and the Jacobi theta functions are

$$
\begin{aligned}
\eta(\tau) & =q^{\frac{1}{24}} \prod_{n=1}^{\infty}\left(1-q^{n}\right) \\
\vartheta_{1}(\tau, z) & =-i q^{\frac{1}{8}} y^{\frac{1}{2}} \prod_{n=1}^{\infty}\left(1-q^{n}\right)\left(1-y q^{n}\right)\left(1-y^{-1} q^{n-1}\right) \\
\vartheta_{2}(\tau, z) & =2 q^{\frac{1}{8}} \cos (\pi z) \prod_{n=1}^{\infty}\left(1-q^{n}\right)\left(1+y q^{n}\right)\left(1+y^{-1} q^{n}\right) \\
\vartheta_{3}(\tau, z) & =\prod_{n=1}^{\infty}\left(1-q^{n}\right)\left(1+y q^{n-1 / 2}\right)\left(1+y^{-1} q^{n-1 / 2}\right) \\
\vartheta_{4}(\tau, z) & =\prod_{n=1}^{\infty}\left(1-q^{n}\right)\left(1-y q^{n-1 / 2}\right)\left(1-y^{-1} q^{n-1 / 2}\right) .
\end{aligned}
$$


Under modular transformations the $\vartheta$ and $\eta$ functions transform as

$$
\begin{array}{ll}
\vartheta_{1}(\tau+1, z)=e^{\frac{2 \pi i}{8}} \vartheta_{1}(\tau, z) & \vartheta_{1}\left(-\frac{1}{\tau}, \frac{z}{\tau}\right)=-(-i \tau)^{\frac{1}{2}} e^{\frac{i \pi z^{2}}{\tau}} \vartheta_{1}(\tau, z), \\
\vartheta_{2}(\tau+1, z)=e^{\frac{2 \pi i}{8}} \vartheta_{2}(\tau, z) & \vartheta_{2}\left(-\frac{1}{\tau}, \frac{z}{\tau}\right)=(-i \tau)^{\frac{1}{2}} e^{\frac{i \pi z^{2}}{\tau}} \vartheta_{4}(\tau, z), \\
\vartheta_{3}(\tau+1, z)=\vartheta_{4}(\tau, z) & \vartheta_{3}\left(-\frac{1}{\tau}, \frac{z}{\tau}\right)=(-i \tau)^{\frac{1}{2}} e^{\frac{i \pi z^{2}}{\tau}} \vartheta_{3}(\tau, z), \\
\vartheta_{4}(\tau+1, z)=\vartheta_{3}(\tau, z) & \vartheta_{4}\left(-\frac{1}{\tau}, \frac{z}{\tau}\right)=(-i \tau)^{\frac{1}{2}} e^{\frac{i \pi z^{2}}{\tau}} \vartheta_{2}(\tau, z),
\end{array}
$$

as well as

$$
\eta(\tau+1)=e^{\frac{2 \pi i}{24}} \eta(\tau) \quad \eta\left(-\frac{1}{\tau}\right)=(-i \tau)^{\frac{1}{2}} \eta(\tau) .
$$

The theta constants $\vartheta_{a}(\tau)$ are defined as $\vartheta_{a}(\tau) \equiv \vartheta_{a}(\tau, z=0)$. The standard weak Jacobi forms $\phi_{0,1}$ and $\phi_{-2,1}$ of index 1 and weight 0 and 2 can be defined as [11]

$$
\phi_{0,1}(\tau, z)=4 \sum_{i=2}^{4} \frac{\vartheta_{i}(\tau, z)^{2}}{\vartheta_{i}(\tau, 0)^{2}}, \quad \phi_{-2,1}(\tau, z)=-\frac{\vartheta_{1}(\tau, z)^{2}}{\eta(\tau)^{6}} .
$$

For completeness we give here the first few terms of the McKay-Thompson series that appear in our analysis

$$
\begin{aligned}
& T_{[8 \mathrm{E}]}=\frac{1}{q}+4 q+2 q^{3}-8 q^{5}-q^{7}+20 q^{9}-2 q^{11}-40 q^{13}+\cdots, \\
& T_{[10 \mathrm{E}]}=\frac{1}{q}+q+2 q^{2}+2 q^{3}-2 q^{4}-q^{5}-4 q^{7}-2 q^{8}+5 q^{9}+2 q^{10}+8 q^{12}+\cdots, \\
& T_{[12 \mathrm{I}]}=\frac{1}{q}+2 q+q^{3}-2 q^{7}-2 q^{9}+2 q^{11}+4 q^{13}+3 q^{15}+\cdots, \\
& T_{[21 \mathrm{~B}]}=\frac{1}{q}-q-q^{2}+q^{3}+2 q^{4}-q^{5}+3 q^{6}-q^{7}-q^{8}-2 q^{9}+q^{11}+\cdots, \\
& T_{[21 \mathrm{D}]}=\frac{1}{q}+5 q+8 q^{2}+16 q^{3}+26 q^{4}+44 q^{5}+66 q^{6}+104 q^{7}+\cdots,
\end{aligned}
$$

see $[17,20]$ for more information about these series.

\section{B Modular forms for $\Gamma_{0}(N)$}

In this section, we will describe a basis of the space $M_{k}\left(\Gamma_{0}(N)\right)$ of modular forms of weight $k$ under $\Gamma_{0}(N)$. Our main references for this section are [21, 22].

The space $M_{k}\left(\Gamma_{0}(N)\right)$ of modular forms of weight $k$ splits into a direct sum

$$
M_{k}\left(\Gamma_{0}(N)\right)=E_{k}\left(\Gamma_{0}(N)\right) \oplus S_{k}\left(\Gamma_{0}(N)\right),
$$

where $S_{k}\left(\Gamma_{0}(N)\right)$ is the space of cusp forms, i.e. forms they vanish at all the cusps $^{8}$ of $\overline{\mathbb{H} / \Gamma_{0}(N)}$. The space $E_{k}\left(\Gamma_{0}(N)\right)$ is defined as the unique subspace satisfying (B.1) that is invariant under the action of all Hecke operators [22].

\footnotetext{
${ }^{8}$ The cusps correspond to $\Gamma_{0}(N)$-orbits in $\mathbb{Q} \cup\{\infty\}$, with $\Gamma_{0}(N)$ acting by fractional linear transformations.
} 
A convenient basis for $E_{k}\left(\Gamma_{0}(N)\right)$ is given by (generalised) Eisenstein series [23]. Let

$$
E_{k}(\tau)=-\frac{B_{k}}{2 k}+\sum_{n=1}^{\infty}\left(\sum_{d \mid n} d^{k-1}\right) q^{n}
$$

be the standard Eisenstein series of weight $k$, where $B_{k}$ are the Bernoulli numbers. For $k>2, k$ even, $E_{k}$ is a modular form of weight $k$ under $\mathrm{SL}(2, \mathbb{Z})$, whereas

$$
E_{2}\left(\frac{a \tau+b}{c \tau+d}\right)=(c \tau+d)^{2} E_{2}(\tau)-\frac{1}{4 \pi i} c(c \tau+d) .
$$

The definition of the Eisenstein series can be generalised to include modular forms under $\Gamma_{0}(N)$. In particular, ${ }^{9}$

$$
\psi^{(N)}=q \frac{\partial}{\partial q} \log \frac{\eta(N \tau)}{\eta(\tau)}=E_{2}(\tau)-N E_{2}(N \tau)
$$

is a modular form of weight 2 under $\Gamma_{0}(N)$. The (generalised) Eisenstein series

$$
E_{k}^{\chi_{m}}(\tau)=\sum_{n=1}^{\infty}\left(\sum_{d \mid n} \overline{\chi_{m}(d)} \chi_{m}(n / d) d^{k-1}\right) q^{n},
$$

where $k$ is even and $\chi_{m}$ is a non-trivial Dirichlet character of modulus $m$, is a modular form of weight $k$ under $\Gamma_{0}\left(m^{2}\right)$. The only cases we need are

$$
E_{k}^{(9)}=E_{k}^{\chi_{3}} \in M_{k}\left(\Gamma_{0}(9)\right), \quad E_{k}^{(16)}=E_{k}^{\chi_{4}} \in M_{k}\left(\Gamma_{0}(16)\right), \quad E_{k}^{(144)}=E_{k}^{\chi_{12}} \in M_{k}\left(\Gamma_{0}(144)\right),
$$

where $\chi_{3}, \chi_{4}$ and $\chi_{12}$ are the primitive Dirichlet characters of modulus 3,4 and 12 , that are uniquely determined by

$$
\chi_{3}(2)=-1, \quad \chi_{4}(3)=-1, \quad \chi_{12}(5)=\chi_{12}(7)=-1 .
$$

In general, the space $S_{k}\left(\Gamma_{0}(N)\right)$ is not generated by Eisenstein series. It is obvious that if $M \mid N$, then $M_{k}\left(\Gamma_{0}(M)\right) \subset M_{k}\left(\Gamma_{0}(N)\right)$. More generally, it is easy to see that if $f \in M_{k}\left(\Gamma_{0}(M)\right)$, then, for any divisor $n$ of $N / M, f(n \tau) \in M_{k}\left(\Gamma_{0}(N)\right)$. For all $M \mid N$ and $n \mid(N / M)$, we define the map

$$
\begin{aligned}
\alpha_{n}: M_{k}\left(\Gamma_{0}(M)\right) & \rightarrow M_{k}\left(\Gamma_{0}(N)\right) \\
f(\tau) & \mapsto \alpha_{n}(f)(\tau)=f(n \tau) .
\end{aligned}
$$

The map $\alpha_{n}$ sends cusp forms to cusp forms and the union of the images $\alpha_{n}\left(S_{k}\left(\Gamma_{0}(N / n)\right)\right) \subseteq$ $S_{k}\left(\Gamma_{0}(N)\right)$, for all $n \mid N, n>1$, is called the old subspace of cusp forms. The complement of the old subspace which is invariant under all Hecke operators is called the new subspace. Thus, we have a decomposition

$$
S_{k}\left(\Gamma_{0}(N)\right)=\bigoplus_{M|N n|(N / M)} \bigoplus_{n} \alpha_{n}\left(S_{k}\left(\Gamma_{0}(M)\right)_{\text {new }}\right)
$$

\footnotetext{
${ }^{9}$ The Eisenstein series $\psi^{(N)}$ are related to the modular forms $\phi^{(N)}$ in $[7]$ by $\psi^{(N)}=\frac{N-1}{24} \phi^{(N)}(\tau)$.
} 
where $S_{k}\left(\Gamma_{0}(M)\right)_{\text {new }}$ is the new subspace for $\Gamma_{0}(M)$. A basis for $S_{k}\left(\Gamma_{0}(M)\right)_{\text {new }}$ for the cases of interest are listed below; a more extended list of their coefficients can be found at http://modi.countnumber.de/. Some Fourier expansions have been computed using SAGE (http://www.sagemath.org/).

- Cusp forms $f_{M} \in S_{2}\left(\Gamma_{0}(M)\right)_{\text {new }}$ - if there is more than one generator, the different generators are denoted by $f_{M, a}, f_{M, b}, \ldots$.

$$
\begin{aligned}
f_{21}(\tau) & =q-q^{2}+q^{3}-q^{4}-2 q^{5}-q^{6}-q^{7}+3 q^{8}+q^{9}+2 q^{10}+\cdots \\
f_{23, a}(\tau) & =q-q^{3}-q^{4}-2 q^{6}+2 q^{7}-q^{8}+2 q^{9}+2 q^{10}+\cdots \\
f_{23, b}(\tau) & =-q^{2}+2 q^{3}+q^{4}-2 q^{5}-q^{6}-2 q^{7}+2 q^{8}+2 q^{10}+\cdots \\
f_{24}(\tau) & =q-q^{3}-2 q^{5}+q^{9}+4 q^{11}-2 q^{13}+2 q^{15}+2 q^{17}+\cdots \\
f_{48}(\tau) & =q+q^{3}-2 q^{5}+q^{9}-4 q^{11}-2 q^{13}-2 q^{15}+2 q^{17}+\cdots \\
f_{63, a}(\tau) & =q+q^{2}-q^{4}+2 q^{5}-q^{7}-3 q^{8}+2 q^{10}-4 q^{11}-2 q^{13}+\cdots \\
f_{63, b}(\tau) & =q+q^{4}+q^{7}-6 q^{10}+2 q^{13}-5 q^{16}+\cdots \\
f_{63, c}(\tau) & =q^{2}-2 q^{5}-q^{8}+2 q^{11}+q^{14}+2 q^{17}+\cdots \\
f_{72}(\tau) & =q+2 q^{5}-4 q^{11}-2 q^{13}-2 q^{17}-4 q^{19}+\cdots \\
f_{144, a}(\tau) & =q+4 q^{7}+2 q^{13}-8 q^{19}-5 q^{25}+4 q^{31}+\cdots \\
f_{144, b}(\tau) & =q+2 q^{5}+4 q^{11}-2 q^{13}-2 q^{17}+4 q^{19}+\cdots
\end{aligned}
$$

- Cusp forms $g_{M} \in S_{4}\left(\Gamma_{0}(M)\right)_{\text {new }}$

$$
\begin{aligned}
g_{5}(\tau) & =q-4 q^{2}+2 q^{3}+8 q^{4}-5 q^{5}-8 q^{6}+6 q^{7}-23 q^{9}+\cdots \\
g_{6}(\tau) & =q-2 q^{2}-3 q^{3}+4 q^{4}+6 q^{5}+6 q^{6}-16 q^{7}-8 q^{8}+9 q^{9}+\cdots \\
g_{8}(\tau) & =q-4 q^{3}-2 q^{5}+24 q^{7}-11 q^{9}-44 q^{11}+22 q^{13}+\cdots \\
g_{10}(\tau) & =q+2 q^{2}-8 q^{3}+4 q^{4}+5 q^{5}-16 q^{6}-4 q^{7}+8 q^{8}+37 q^{9}+\cdots \\
g_{12}(\tau) & =q+3 q^{3}-18 q^{5}+8 q^{7}+9 q^{9}+36 q^{11}-10 q^{13}-54 q^{15}+\cdots .
\end{aligned}
$$

- Cusp forms $h_{M} \in S_{6}\left(\Gamma_{0}(M)\right)_{\text {new }}$

$$
\begin{aligned}
h_{3}(\tau) & =q-6 q^{2}+9 q^{3}+4 q^{4}+6 q^{5}-54 q^{6}-40 q^{7}+168 q^{8}+\cdots \\
h_{7, a}(\tau) & =q-10 q^{2}-14 q^{3}+68 q^{4}-56 q^{5}+140 q^{6}-49 q^{7}-360 q^{8}+\cdots \\
h_{7, b}(\tau) & =q+4 q^{2}-2 q^{4}-14 q^{5}-84 q^{6}+49 q^{7}-10 q^{8}+\cdots \\
h_{7, c}(\tau) & =q^{2}-6 q^{3}+9 q^{4}+10 q^{5}-30 q^{6}+11 q^{8}+\cdots \\
h_{21, a}(\tau) & =q+q^{2}-9 q^{3}-31 q^{4}-34 q^{5}-9 q^{6}-49 q^{7}-63 q^{8}+\cdots \\
h_{21, b}(\tau) & =q+5 q^{2}+9 q^{3}-7 q^{4}+94 q^{5}+45 q^{6}-49 q^{7}-195 q^{8}+\cdots \\
h_{21, c}(\tau) & =q-6 q^{2}-9 q^{3}+4 q^{4}+78 q^{5}+54 q^{6}+49 q^{7}+168 q^{8}+\cdots \\
h_{21, d}(\tau) & =q+10 q^{2}+9 q^{3}+68 q^{4}-106 q^{5}+90 q^{6}-49 q^{7}+360 q^{8}+\cdots .
\end{aligned}
$$

- Cusp forms $k_{M} \in S_{8}\left(\Gamma_{0}(M)\right)_{\text {new }}$

$$
k_{2}(\tau)=q-8 q^{2}+12 q^{3}+64 q^{4}-210 q^{5}-96 q^{6}+1016 q^{7}-512 q^{8}+\cdots .
$$


- Cusp forms $l_{M} \in S_{12}\left(\Gamma_{0}(M)\right)_{\text {new }}$

$$
\begin{aligned}
l_{3}(\tau) & =q+78 q^{2}-243 q^{3}+4036 q^{4}-5370 q^{5}-18954 q^{6}-27760 q^{7}+\cdots \\
l_{6, a}(\tau) & =q+32 q^{2}+243 q^{3}+1024 q^{4}+3630 q^{5}+7776 q^{6}+32936 q^{7}+\cdots \\
l_{6, b}(\tau) & =q-32 q^{2}-243 q^{3}+1024 q^{4}+5766 q^{5}+7776 q^{6}+72464 q^{7}+\cdots \\
l_{6, c}(\tau) & =q-32 q^{2}+243 q^{3}+1024 q^{4}-11730 q^{5}-7776 q^{6}-50008 q^{7}+\cdots .
\end{aligned}
$$

To summarise, $M_{k}\left(\Gamma_{0}(N)\right)$ is thus generated by

$$
\begin{array}{lll}
k=2: & \psi^{(n)}(\tau) & \text { for } n \mid N, n>1 \\
& E_{2}^{\chi_{m}}(n \tau) & \text { for } n m^{2} \mid N, m>1 \\
& f_{M, a}(n \tau), f_{M, b}(n \tau), \ldots & \text { for } n M \mid N \\
k=4: & E_{4}(n \tau) & \\
& E_{4}^{\chi_{m}}(n \tau) & \text { for } n \mid N \\
& g_{M, a}(n \tau), g_{M, b}(n \tau), \ldots & \text { for } n m^{2} \mid N, m>1 \\
& \text { for } n M \mid N,
\end{array}
$$

and the cases $k=6,8, \ldots$ etc. are similar to $k=4$, with $g_{M, a}$ replaced by $h_{M, a}$, etc.

\section{The modular forms of weight two}

With the conventions of the previous appendix we can now give the explicit expressions for the modular forms $F_{g} \in M_{2}\left(\Gamma_{0}(N h)\right)$, as well as for $F_{g}^{h} \in M_{2 h}\left(\Gamma_{0}(N)\right)$. For the classes in (3.2) for which $h=1$, the relevant formulae are [7]

$$
\begin{aligned}
F_{2 \mathrm{~A}}(\tau) & =16 \psi^{(2)}(\tau) \\
F_{3 \mathrm{~A}}(\tau) & =9 \psi^{(3)}(\tau) \\
F_{4 \mathrm{~B}}(\tau) & =-4 \psi^{(2)}(\tau)+8 \psi^{(4)}(\tau) \\
F_{5 \mathrm{~A}}(\tau) & =5 \psi^{(5)}(\tau) \\
F_{6 \mathrm{~A}}(\tau) & =-2 \psi^{(2)}(\tau)-3 \psi^{(3)}(\tau)+6 \psi^{(6)}(\tau) \\
F_{7 \mathrm{AB}}(\tau) & =\frac{7}{2} \psi^{(7)}(\tau) \\
F_{8 \mathrm{~A}}(\tau) & =-2 \psi^{(4)}(\tau)+4 \psi^{(8)}(\tau) \\
F_{11 \mathrm{~A}}(\tau) & =\frac{11}{5}\left(\psi^{(11)}(\tau)-\eta(\tau)^{2} \eta(11 \tau)^{2}\right) \\
F_{14 \mathrm{AB}}(\tau) & =\frac{1}{6}\left(-2 \psi^{(2)}(\tau)-7 \psi^{(7)}(\tau)+14 \psi^{(14)}(\tau)-14 \eta(\tau) \eta(2 \tau) \eta(7 \tau) \eta(14 \tau)\right) \\
F_{15 \mathrm{AB}}(\tau) & =\frac{1}{8}\left(-3 \psi^{(3)}(\tau)-5 \psi^{(5)}(\tau)+15 \psi^{(15)}(\tau)-15 \eta(\tau) \eta(3 \tau) \eta(5 \tau) \eta(15 \tau)\right) \\
F_{23 \mathrm{AB}} & =\frac{23}{22}\left(\psi^{(23)}(\tau)-f_{23, a}(\tau)+3 f_{23, b}(\tau)\right)
\end{aligned}
$$

In the remaining cases we have found - the formulae for $F_{2 \mathrm{~B}}$ and $F_{4 \mathrm{~A}}$ were already obtained in $[7]$ 


\begin{tabular}{|c|ccccccc|}
\hline$p$ & 1 & 2 & 3 & 4 & 5 & 6 & 7 \\
\hline$c_{p}$ & $\frac{1}{22951565}$ & $-\frac{4096}{22951565}$ & $-\frac{531441}{22951565}$ & $\frac{2176782336}{22951565}$ & $-\frac{189277}{58735}$ & $-\frac{91776}{3455}$ & $\frac{37712628}{58735}$ \\
\hline \hline$p$ & 8 & 9 & 10 & 11 & 12 & 13 & \\
\hline$c_{p}$ & $\frac{27713664}{3455}$ & $-\frac{297}{140}$ & $-\frac{308}{145}$ & $-\frac{95}{52}$ & $-\frac{194697}{71978}$ & $\frac{304128}{2117}$ & \\
\hline
\end{tabular}

Table 5. Coefficients $c_{p}$ in the expansion of $F_{6 \mathrm{~B}}(\tau)^{6}$.

- $g=2 \mathrm{~B}, h=2$

$$
\begin{aligned}
F_{2 \mathrm{~B}}(\tau) & =-24 \psi^{(2)}(\tau)+16 \psi^{(4)}(\tau) & & \in M_{2}\left(\Gamma_{0}(4)\right) \\
F_{2 \mathrm{~B}}(\tau)^{2} & =-16 E_{4}(\tau)+256 E_{4}(2 \tau) & & \in M_{4}\left(\Gamma_{0}(2)\right)
\end{aligned}
$$

- $g=3 \mathrm{~B}, h=3$

$$
\begin{array}{clrl}
F_{3 \mathrm{~B}}(\tau) & =-6 \psi^{(3)}(\tau)+\frac{9}{2} \psi^{(9)}(\tau)-\frac{9}{2} E_{2}^{(9)}(\tau) & \in M_{2}\left(\Gamma_{0}(9)\right) \\
F_{3 \mathrm{~B}}(\tau)^{3}=-1944 \psi^{(3)}(\tau)^{3}+\frac{45}{2} E_{6}(\tau)-\frac{2187}{2} E_{6}(3 \tau) & \in M_{6}\left(\Gamma_{0}(3)\right)
\end{array}
$$

- $g=4 \mathrm{~A}, h=2$

$$
\begin{aligned}
F_{4 \mathrm{~A}}(\tau) & =4 \psi^{(2)}(\tau)-12 \psi^{(4)}(\tau)+8 \psi^{(8)}(\tau) & & \in M_{2}\left(\Gamma_{0}(8)\right) \\
F_{4 \mathrm{~A}}(\tau)^{2} & =-16 E_{4}(2 \tau)+256 E_{4}(4 \tau) & & \in M_{4}\left(\Gamma_{0}(4)\right)
\end{aligned}
$$

- $g=4 \mathrm{C}, h=4$

$$
\begin{array}{cl}
F_{4 \mathrm{C}}(\tau)=2 \psi^{(4)}(\tau)-6 \psi^{(8)}(\tau)+4 \psi^{(16)}(\tau)-4 E_{2}^{(16)}(\tau) & \in M_{2}\left(\Gamma_{0}(16)\right) \\
F_{4 \mathrm{C}}(\tau)^{4}=-\frac{32}{17} E_{8}(2 \tau)+\frac{8192}{17} E_{8}(4 \tau)-16 k_{2}(\tau)-\frac{512}{17} k_{2}(2 \tau) & \in M_{8}\left(\Gamma_{0}(4)\right)
\end{array}
$$

- $g=6 \mathrm{~B}, h=6$

$$
\begin{aligned}
& F_{6 \mathrm{~B}}(\tau)=-\frac{3}{2} \psi^{(2)}(\tau)-2 \psi^{(3)}(\tau)+\psi^{(4)}(\tau)+6 \psi^{(6)}(\tau)+\frac{3}{2} \psi^{(9)}(\tau)-4 \psi^{(12)}(\tau) \\
& -\frac{9}{2} \psi^{(18)}(\tau)+3 \psi^{(36)}(\tau)-\frac{3}{2} E_{2}^{(9)}(\tau) \\
& -9 E_{2}^{(9)}(2 \tau)-12 E_{2}^{(9)}(4 \tau) \quad \in M_{2}\left(\Gamma_{0}(36)\right) \\
& F_{6 \mathrm{~B}}(\tau)^{6}=c_{1} E_{12}(\tau)+c_{2} E_{12}(2 \tau)+c_{3} E_{12}(3 \tau)+c_{4} E_{12}(6 \tau)+c_{5} \Delta(\tau) \\
& +c_{6} \Delta(2 \tau)+c_{7} \Delta(3 \tau)+c_{8} \Delta(6 \tau)+c_{9} l_{6, a}(\tau) \\
& +c_{10} l_{6, b}(\tau)+c_{11} l_{6, c}(\tau)+c_{12} l_{3}(\tau)+c_{13} l_{3}(2 \tau) \quad \in M_{12}\left(\Gamma_{0}(6)\right)
\end{aligned}
$$

where $\Delta(\tau)=\left(\left(240 E_{4}(\tau)\right)^{3}-\left(504 E_{6}(\tau)^{2}\right)\right) / 1728$ and the coefficients $c_{p}$ are given in table 5 . 
- $g=10 \mathrm{~A}, h=2$

$$
\begin{aligned}
F_{10 \mathrm{~A}}(\tau)= & \psi^{(2)}(\tau)-\frac{2}{3} \psi^{(4)}(\tau)+\frac{5}{3} \psi^{(5)}(\tau)-5 \psi^{(10)}(\tau) \\
& +\frac{10}{3} \psi^{(20)}(\tau)-\frac{10}{3} \eta(2 \tau)^{2} \eta(10 \tau)^{2} \\
F_{10 \mathrm{~A}}(\tau)^{2}= & \frac{1}{39} E_{4}(\tau)-\frac{16}{39} E_{4}(2 \tau)-\frac{625}{39} E_{4}(5 \tau)+\frac{10000}{39} E_{4}(10 \tau) \\
& -\frac{10}{3} g_{10}(\tau)-\frac{35}{13} g_{5}(\tau)+\frac{40}{13} g_{5}(2 \tau)
\end{aligned}
$$

- $g=12 \mathrm{~A}, h=2$

$$
\begin{aligned}
F_{12 \mathrm{~A}}(\tau)= & -\frac{1}{2} \psi^{(2)}(\tau)+\frac{3}{2} \psi^{(4)}(\tau)+\frac{3}{2} \psi^{(6)}(\tau)-\psi^{(8)}(\tau) \\
& -\frac{9}{2} \psi^{(12)}(\tau)+3 \psi^{(24)}(\tau)-3 f_{24}(\tau) \\
F_{12 \mathrm{~A}}(\tau)^{2}= & \frac{1}{5} E_{4}(2 \tau)-\frac{16}{5} E_{4}(4 \tau)-\frac{81}{5} E_{4}(6 \tau)+\frac{1296}{5} E_{4}(12 \tau) \\
& -3 g_{12}(\tau)-3 g_{6}(\tau)+\frac{24}{5} g_{6}(2 \tau)
\end{aligned}
$$

- $g=12 \mathrm{~B}, h=12$

$$
\begin{aligned}
F_{12 \mathrm{~B}}(\tau)= & \frac{1}{8} \psi^{(4)}(\tau)-\frac{3}{8} \psi^{(8)}(\tau)-\frac{1}{2} \psi^{(12)}(\tau)+\frac{1}{4} \psi^{(16)}(\tau)+\frac{3}{2} \psi^{(24)}(\tau)+\frac{3}{8} \psi^{(36)}(\tau) \\
& -\psi^{(48)}(\tau)-\frac{9}{8} \psi^{(72)}(\tau)+\frac{3}{4} \psi^{(144)}(\tau)-\frac{3}{2} E_{2}^{(9)}(4 \tau)-9 E_{2}^{(9)}(8 \tau)-12 E_{2}^{(9)}(16 \tau) \\
& -\frac{1}{4} E_{2}^{(16)}(\tau)-3 E_{2}^{(16)}(3 \tau)-\frac{27}{4} E_{2}^{(16)}(9 \tau)-\frac{3}{4} E_{2}^{(144)}(\tau)-\frac{3}{2} f_{24}(2 \tau) \\
& -\frac{27}{2} f_{24}(6 \tau)-\frac{3}{4} f_{48}(\tau)+\frac{27}{4} f_{48}(3 \tau)+\frac{9}{2} f_{72}(2 \tau)-\frac{9}{4} f_{144, b}(\tau) \in M_{2}\left(\Gamma_{0}(144)\right)
\end{aligned}
$$

We have also worked out the expansion of $F_{12 \mathrm{~B}}^{12}$ in terms of generators of $M_{24}\left(\Gamma_{0}(12)\right)$, but since the final expression is exceedingly complicated, we shall not spell it out here.

- $g=21 \mathrm{AB}, h=3$

$$
\begin{aligned}
F_{21 \mathrm{AB}}(\tau)= & \frac{1}{8} \psi^{(3)}(\tau)+\frac{7}{32} \psi^{(7)}(\tau)-\frac{3}{32} \psi^{(9)}(\tau)-\frac{7}{8} \psi^{(21)}(\tau)+\frac{21}{32} \psi^{(63)}(\tau)+\frac{3}{32} E_{2}^{(9)}(\tau) \\
& -\frac{147}{32} E_{2}^{(9)}(7 \tau)-\frac{21}{32} f_{21}(\tau)+\frac{189}{32} f_{21}(3 \tau)-\frac{63}{32} f_{63, a}(\tau) \quad \in M_{2}\left(\Gamma_{0}(63)\right) \\
F_{21 \mathrm{AB}}(\tau)^{3}= & -\frac{1}{169936} E_{6}(\tau)+\frac{729}{169936} E_{6}(3 \tau)+\frac{117649}{169936} E_{6}(7 \tau)-\frac{85766121}{169936} E_{6}(21 \tau) \\
& -\frac{85641}{36608} h_{3}(\tau)-\frac{352947}{36608} h_{3}(7 \tau)-\frac{64491}{94600} h_{7 a}(\tau)-\frac{11263}{9728} h_{7 b}(\tau)-\frac{18277}{4864} h_{7 c}(\tau) \\
& -\frac{2740311}{189200} h_{7 a}(3 \tau)+\frac{66339}{9728} h_{7 b}(3 \tau)-\frac{250047}{4864} h_{7 c}(3 \tau)-\frac{441}{352} h_{21 a}(\tau) \\
& -\frac{1323}{8800} h_{21 b}(\tau)-\frac{735}{512} h_{21 c}(\tau)-\frac{3087}{6400} h_{21 d}(\tau) \quad \in M_{6}\left(\Gamma_{0}(21)\right) .
\end{aligned}
$$




\section{References}

[1] T. Eguchi, H. Ooguri and Y. Tachikawa, Notes on the K3 Surface and the Mathieu group $M_{24}$, arXiv: 1004.0956 [SPIRES].

[2] T. Eguchi and K. Hikami, Superconformal Algebras and Mock Theta Functions 2. Rademacher Expansion for K3 Surface, arXiv:0904.0911 [SPIRES].

[3] I.B. Frenkel, J. Lepowsky and A. Meurman, Vertex Operator Algebras and the Monster, Pure and Applied Math. Vol. 134, Academic Press, Boston U.S.A. (1988).

[4] J.G. Thompson, Some numerology between the Fischer-Griess Monster and the elliptic modular function, Bull. Lond. Math. Soc. 11 (1979) 352.

[5] J.H. Conway and S. Norton, Monstrous moonshine, Bull. Lond. Math. Soc. 11 (1979) 308.

[6] T. Gannon, Moonshine beyond the Monster: The Bridge connecting Algebra, Modular Forms and Physics, Cambridge University Press, Cambridge U.K. (2006).

[7] M.C.N. Cheng, K3 Surfaces, $N=4$ Dyons and the Mathieu Group $M_{24}$, arXiv:1005.5415 [SPIRES].

[8] M.R. Gaberdiel, S. Hohenegger and R. Volpato, Mathieu twining characters for K3, JHEP 09 (2010) 058 [arXiv: 1006.0221] [SPIRES].

[9] T. Kawai, Y. Yamada and S.-K. Yang, Elliptic genera and $N=2$ superconformal field theory, Nucl. Phys. B 414 (1994) 191 [hep-th/9306096] [SPIRES].

[10] J.R. David, D.P. Jatkar and A. Sen, Product representation of dyon partition function in CHL models, JHEP 06 (2006) 064 [hep-th/0602254] [SPIRES].

[11] M. Eichler and D. Zagier, The Theory of Jacobi Forms, Birkhäuser, Basel Swizerland (1985).

[12] T. Eguchi, H. Ooguri, A. Taormina and S.-K. Yang, Superconformal Algebras and String Compactification on Manifolds with SU(N) Holonomy, Nucl. Phys. B 315 (1989) 193 [SPIRES].

[13] T. Eguchi and A. Taormina, Character formulas for the $\mathcal{N}=4$ superconformal algebra, Phys. Lett. B 200 (1988) 315 [SPIRES].

[14] T. Eguchi and A. Taormina, On the unitary representations of $N=2$ and $N=4$ superconformal algebras, Phys. Lett. B 210 (1988) 125 [SPIRES].

[15] S. Mukai, Finite groups of automorphisms of K3 surfaces and the Mathieu group, Invent. Math. 94 (1988) 183.

[16] S. Kondõ, Niemeier lattices, Mathieu groups and finite groups of symplectic automorphisms of K3 surfaces, Duke Math. J. 92 (1998) 593.

[17] D. Ford, J. McKay and S. Norton, More on replicable functions, Commun. Algebra 22 (1994) 5175.

[18] A. Taormina and K. Wendland, The symmetries of the tetrahedral Kummer surface in the Mathieu group $M_{24}$, arXiv:1008.0954 [SPIRES].

[19] M.R. Gaberdiel, S. Hohenegger and R. Volpato, work in progress.

[20] http://www.research.att.com/ njas/sequences/.

[21] S. Lang, Introduction to Modular Forms, Grundlehren der Mathematischen Wissenschaften. Vol. 222, Springer Verlag, Berlin Germany (1995). 
[22] W. Stein, Modular Forms, a Computational Approach, Graduate Studies in Mathematics. Vol. 79, American Mathematical Society, Providence U.S.A. (2007).

[23] T. Miyake, Modular Forms, Springer Verlag, Berlin Germany (2006).

[24] T. Eguchi and K. Hikami, Note on Twisted Elliptic Genus of K3 Surface, arXiv:1008.4924 [SPIRES]. 